\title{
A Framework for the Image Retrieval System Based on Histogram Normalization Technique with Python

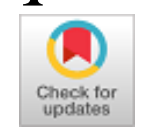

\author{
Madhuri Pydi, K.L.Sailaja
}

\begin{abstract}
The processing of multimedia content is used for real-world computer vision in various applications, and digital images make up a large part of multimedia data. The processing of multimedia content is used for real-computer vision in various applications, and digital images make up a large part of multimedia data. Content-based Retrieval of photographs (CBIR) is a system of picture recovery which utilizes the visual highlights of a picture, for example, shading, shape and surface so as to look through the client based inquiry pictures from the huge databases. CBIR relies upon highlight extraction of a picture which are the visual highlights and these highlights are extricated naturally i.e. without human collaboration. We intend in this paper to provide a detailed overview of recent developments related to CBIR and image representation. We researched the main aspects of various models of image recovery and image representation from low-level feature extraction to recent semantict ML approaches. And, for extraction of features, HSV, image segmentation and color histogram techniques are used, which effectively gives us the main point in an image that these techniques are used to minimize complexity, expense, and energy and time consumption. Then a machine learning model is trained for similarity test and the validation and texting phases are performed accordingly which leads to better performance as. Then a machine learning model is trained for similarity testing and then the validation and texting steps are performed accordingly, resulting in improved results compared to previously performed techniques. The precision values in the proposed technique are fairly excellent.
\end{abstract}

Keywords: CBIR, Feature Extraction, HSV, Image segmentation, Multimedia.

\section{INTRODUCTION}

The growth in computing power and electronic storage space has contributed to an exponential rise in the amount of multimedia content in the form of photographs and videos accessible to consumers. But searching for relevant information requires a strong retrieval system. Image retrieval techniques can be of three types: Meta-data search: text-based tag search and image-embedded annotations. Search by Example: Search based on the image's unique content such as Color, Texture, and Shapes. The systems using this technique are called CBIR systems. Hybrid

Revised Manuscript Received on April 25, 2020.

* Correspondence Author

Madhuri pydi*, from Computer Science and Engineering, VR Siddhartha Engineering College, Vijayawada, India. Email: madhupydi1996@gmail.com

K.L.Sailaja, Department of CSE, from VR Siddhartha Engineering College, Vijayawada, India. Email: sailaja0905@gmail.com

(C) The Authors. Published by Blue Eyes Intelligence Engineering and Sciences Publication (BEIESP). This is an open access article under the CC BY-NC-ND license (http://creativecommons.org/licenses/by-nc-nd/4.0/) approach: The two approaches merged above. Although searching by metadata is by far the easiest of all methods, it does have significant drawbacks. In a program focused on metadata-only scanning, the individual image itself is rarely examined. Many times the images are tagged or annotated incorrectly and thus produce irrelevant results. Also, many images don't contain any textual information embedded in them. Even if they do, the picture description is based on human interference in the database. CBIR-based models, however, depend entirely on the image's intrinsic features and are therefore incapable of any human error.

Owing to the late progress in innovation, the use of digital cameras, smartphones and the Internet is growing. Any image recovery model's primary need is to look at and mastermind the images that are in a visual semantic relationship with the client's given query. The greater part of the web crawlers on the Internet recover the pictures based on content based methodologies that require subtitles as info. The client presents an inquiry by entering some content or catchphrases that are coordinated with the watchwords that are put in the document. The yield is generated based on coordination in catchphrases, and this procedure will recover the non-applicable images. The differentiation in visual observation of humans and the manual naming / explication is the fundamental intent behind the superfluous yield.

\section{RELATED WORK}

Meenaakshi N et.al. [1] MPEG-7 and Edge Directivity Descriptor is utilized to take out the component vectors of a specific picture from the picture database. A compelling graphical UI with the basic utility with the end goal of execution of various pictures as picture exhibition talked about. In this way, the essential thought of this strategy is to grow such kind of substance based picture recovery framework that can place into activity in a huge picture display work area application to allow able perusing with the assistance of two distinctive investigation techniques individually. There are number of catchphrases and different metadata were used to deal with the pictures and recordings in the enormous database in early years. Osman Emre Dai et.al., [2] Proposed a technique it considers a novel proportion of name probability in the structure of inadequate remaking based classifiers and sums up the first scanty classifier to the case both single-name and multilabel RS picture recovery issues. At long last, to improve recovery execution, we acquaint a technique with abuse the affectability of the meager recreation based strategy to various lexicon words.

Published By:

Blue Eyes Intelligence Engineering \& Sciences Publication 2300 (C) Copyright: All rights reserved.

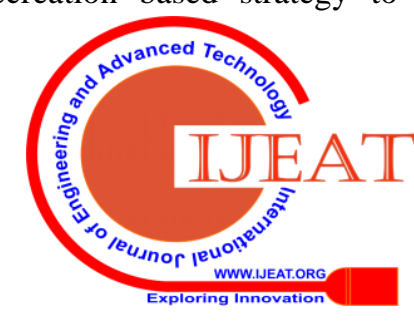


Test results got on two benchmark chronicles show the adequacy of the proposed framework. They present a novel substance based remote detecting (RS) picture recovery framework that comprises of the accompanying. Initial, a picture depiction technique that describes both spatial and ghostly data substance of RS pictures. Second, an administered recovery strategy that effectively models and endeavors the sparsity of RS picture descriptors.

Content-based picture recovery utilizing fluffy class participation and rules dependent on classifier certainty proposed by Jatindra Kumar Das et.al. [3], this review proposes a new recovery plot for the scene. The general investigation period of the late proposed solution called 'class participation-based recovery' (CMR) is now to be that. The proposed strategy distinguishes the trust in the order and constrains the inquiry to single yield class and along these lines, lessens the general hunt time by $21.76 \%$ when contrasted with CMR.

Licheng Jiao et.al. [4] Proposed strategy can be inserted into any EO mining frameworks to assist them with finishing the EO missions. In the wake of contrasting the technique introduced right now others, it is obvious that the strategy performs more adequately than others from the CBIR viewpoint. In view of the semantic classification and district based closeness measure, a novel manufactured gap radar (SAR) picture recovery strategy is proposed right now, is motivated by the current substance based picture recovery (CBIR) strategies.

Jing-Ming Guo et.al. [5] Presents a method for Content-Based Image Retrieval (CBIR) by abusing the upside of low multifaceted nature Ordered-Dither Block Truncation Coding for the age of picture content descriptor. In encoding step, ODBTC packs a picture hinder into relating quantizes and bitmap picture. Two picture highlights are proposed to file a picture, to be specific Color Co-event Feature and Bit Pattern Features, which are produced legitimately from ODBTC encoded information streams without playing out the disentangling procedure.

Jose Ramos et.al. [6] Proposed another system that naturally learns the comparability between the few tests from literary separations removed from radiology reports, consequently effectively decreasing the quantity of comments required. The strategy right off the bat induces the connection between patients by utilizing data recovery systems to decide the literary separations between understanding radiology reports. These separations are in this manner used to manage a measurement learning calculation that changes the picture space as needs be to printed separations. CBIR frameworks with various picture portrayals and various degrees of clinical explanations were assessed, with and without supervision from printed separations, utilizing a database of PC tomography sweeps of patients with interstitial lung sicknesses.

Jorge Sevilla et.al. [7] Present another computationally effective CBIR framework for hyper phantom information) which utilizes meager unmixing ideas to recover hyper ghostly scenes, in view of their substance, from enormous archives. The inquiry is directed by an unearthly library which is used as a manual for vigorously and effectively recovering the information. Given the enormous size of the libraries and the sparse unmixing arrangements, the combination of sparse unmixing with the CBIR engine provides crucial focal points.

Guo Jing-Ming et al. [8] presents a different way of handling file shading images using highlights isolated from the Error Diffusion Block Truncation Coding. The EDBTC generates two quantizing shades and a bitmap image that are both treated using Vector Quantization to construct the descriptor image. Thus, two highlights are displayed, in particular the Color Histogram feature and the Bit Pattern Histogram function, in order to gage the comparability between a query image and the objective image in the database. CHF and BHF are processed separately from the shading quantize specified in VQ and the bitmap image filed in VQ.

Yibing Ma et.al. [9] Proposed a solo, exact and quick recovery strategy for bosom histopathological picture. In particular, the strategy presents neighborhood factual component of cores for morphology and circulation of cores, and utilizes Gabor highlight to depict surface data. Inert Dirichlet Allocation model is used for elevated level semantic mining.

Shikui We et.al. [10] Proposed a system has two streams that all the while handle two assignments. The standard spotlights on removing discriminative visual highlights that are firmly identified with semantic qualities. Then, the assistant stream plans to encourage the standard by diverting the component extraction to the striking picture content that a human may focus on.

\section{METHODOLOGY}

For CBIR and image classification-based models, the high-level image visuals are depicted in the form of feature vectors consisting of numerical values. The work shows that there is a significant difference between perception of the picture function and visual comprehension of humans. Because of this, the research discussed in this field focuses on reducing the semantic gap between describing the role of the image and comprehension of human visuals. Content Based Image Retrieval (CBIR) systems rely on the inherent features of the image, such as color, texture and form. In this section of proposed approach you describe the structure of the proposed approach.

\section{These are the basic steps in CBIR:}

Build the Image Database: for all images, this database is the storehouse. The images pertinent to the query image (the machine input) are to be retrieved from this database. The images may be of any format file (.jpeg, .png, etc)

Feature extraction function: Each image must be represented by its respective vector function. This function vector will be an array of numbers which will contain all the important image data. Then, these vectors will be stored for further use in a database. However, the same extraction method of the function should also be used for the image query.

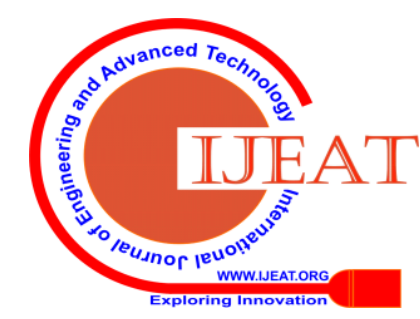


Similarity Comparison: Once the query image's feature vector is created, it is compared to the feature vectors of all the images in the database. This relation is possible with distance functions. The smaller the distance between two vectors of features, the more similar will be the corresponding images. Popular distance features include the Euclidean, Manhattan distance, etc.

Finally show the result: Extract and view the most important images.

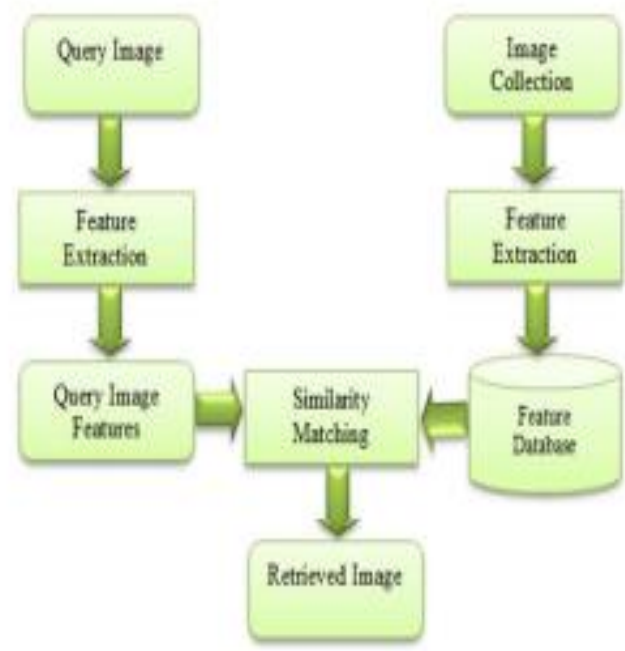

Fig 1. Proposed Methodology

The methodology consists of following phases they are,

- Image Data Set

- Feature Extraction

- Histogram Normalization

- Image Segmentation

- Similarity Matching

- Retrieved Image

\section{A. Image Data Set}

We used Corel-10k as the dataset for this project. This dataset contains 10,000 photos from various contents such as sunset, beach, flower, houses, cars, horses, mountains, fish, fruit, bear etc. There are 100 categories in total and each of those categories includes 100 images in JPEG format.

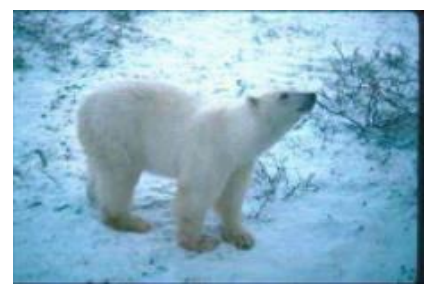

Fig 2. Sample Input Image

\section{B. Feature Extraction}

The proposed CBIR systems, various low-level features of an image are used to achieve the desired results. These are

- Color

- Shape

- Texture

The research is focused on the Color function and has been used in the CBIR framework to describe an image. But, here, we're addressing it widely.

\section{a. Color}

Color is an incredible descriptor that streamlines object distinguishing proof, furthermore, is one of the most as often as possible utilized visual highlights for content-based picture recovery. A few shading spaces, for example, RGB, HSV and a lot more have been produced for various purposes. Despite the fact that there is no concession to which shading space is the best for CBIR, a fitting shading framework is required to guarantee perceptual uniformity. The RGB shading space, a generally utilized framework for speaking to shading pictures, can't for CBIR in light of the fact that it is a perceptually non-uniform and gadget subordinate framework. The most regularly utilized strategy to speak to shading highlight of a picture is the shading histogram.

\section{b. Color Histograms}

Color Histograms are a very accurate and efficient way to compare the similarity between two pictures. They provide relatively accurate information about the distribution of colors in a picture. RGB \& HSV histograms are created to the given test image. However, if not taken care of, there are two major issues which can lead to unexpected outcomes. With Histogram Normalization, the first problem can be solved, and the second with Image Segmentation and Local Color Histograms (LCH) computing.

\section{Histogram Normalization}

Even though the concept of histogram normalization is very basic, it is so successful that it is considered one of the most important steps in CBIR pre-processing. The reason for this method is that even though two physically identical images have very different dimensions (height and width), the ratio of the pixel no of the color histogram to the total number of pixels in a specific bin.

If $n$ is the number of pixels in the color histogram in a specific bin, and $\mathrm{T}$ is the total number of pixels in the image, then for all image resolutions.

$$
\mathrm{n} \div \mathrm{T}=\text { Constant }
$$

And the value in each bin is divided by the total number of pixels in the image while the color histogram is computed. As such, a comparison of histograms (after normalization) is invariant to image scaling.

\section{Image Segmentation}

Rather than registering a HSV shading histogram for the whole picture, we processed a HSV shading histogram for various districts of the picture. Utilizing a worldwide histogram we would be not able to decide where in the picture the specific shading happens. Rather, we would simply realize that there exists some level of that shading in the picture. To cure this issue, we can register shading histograms in areas of the picture. Utilizing areas based histograms rather than worldwide histograms permits us to reenact area in a shading conveyance. For the image descriptor, we are going to divide the image into five different regions:

Published By:

Blue Eyes Intelligence Engineering \&

Retrieval Number: D9060049420/2020CBEIESP

DOI: 10.35940/ijeat.D9060.049420

Journal Website: www.ijeat.org

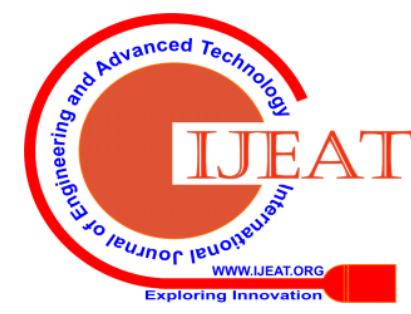


- The top-left corner

- The top-right corner

- The bottom-right corner

- The bottom-left corner

- The center of the image

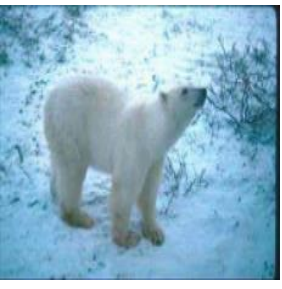

Fig 3. Original Image

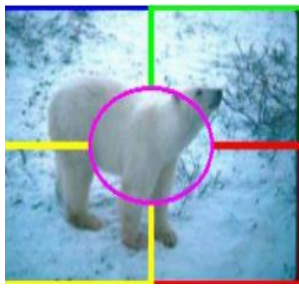

Fig 3.1. Segmented Image
After picture division, the shading histograms for all the five areas are processed lastly consolidated into a solitary list. By tests we discovered that utilizing 16 receptacles for Hue, 7 containers for Saturation and 7 canisters for Value yield the best outcomes. So each picture is spoken to by a component vector which contains 16 × 7 × 7 × $5=3920$ qualities. At long last we standardize the consolidated component vector by separating every one of its qualities with the all-out no of pixels in the picture.

\section{E. Similarity Comparison}

Upon obtaining the query image, all the three steps above are applied to the query image to transform the image into its corresponding function vector. We play with various distance functions and eventually agree to use the Euclidean distance as the metric of similarity between the two characteristic vectors. We sort them out based on the similarity after figuring out this difference between the query image and all the other images in the dataset. The less the distance between two vectors of features the greater the resemblance between the two images that correspond. Finally we display from this list the top 10 related (similar) photos.

\section{F. Retrieved Image}

In this section, we get result of query image respectively, we search for the given query image in the specified data set and get the top 10 relevant images. The result of content based image retrieval system as shown in results and discussion section.

\section{RESULT AND DISCUSSION}

In this section, we discuss about the result of the content based image retrieval system.

\section{The GUI (Graphical user interface):}

Using the Tkinter kit, we created a Graphical User Interface (GUI) in Python. It has 3 buttons:

- Generate Feature Vectors: Generate feature vectors for a specific image sub-set. Within the two textboxes which accompany this button, the first \& last image number can be defined. This feature is designed to allow users to search within a specified range of images and not the entire database.

- Upload image: upload the query image
- $\quad$ Search images: search for the given query image in the specified dataset and view the top 10 relevant results.

- $\quad$ Clear images: Removes all Interface files.

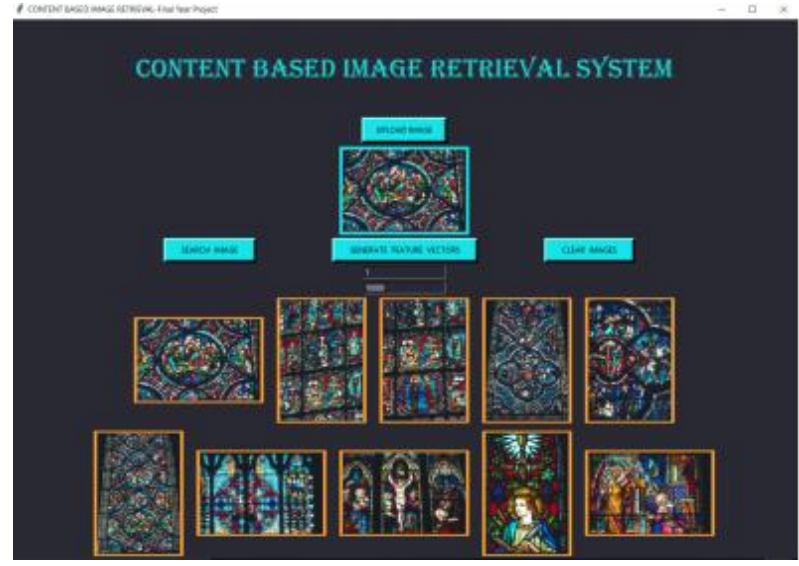

Fig 4. Relevant images for "Painting" category

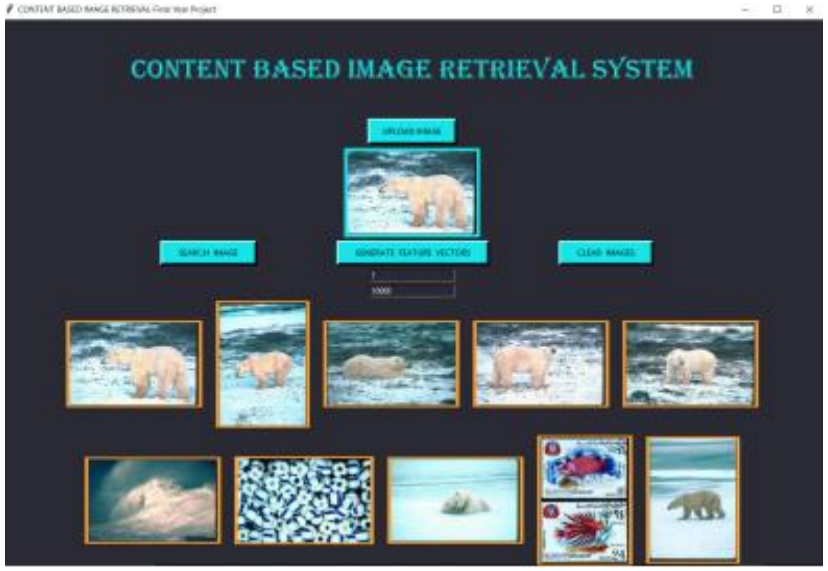

Fig 5. Relevant images for "Bear" category

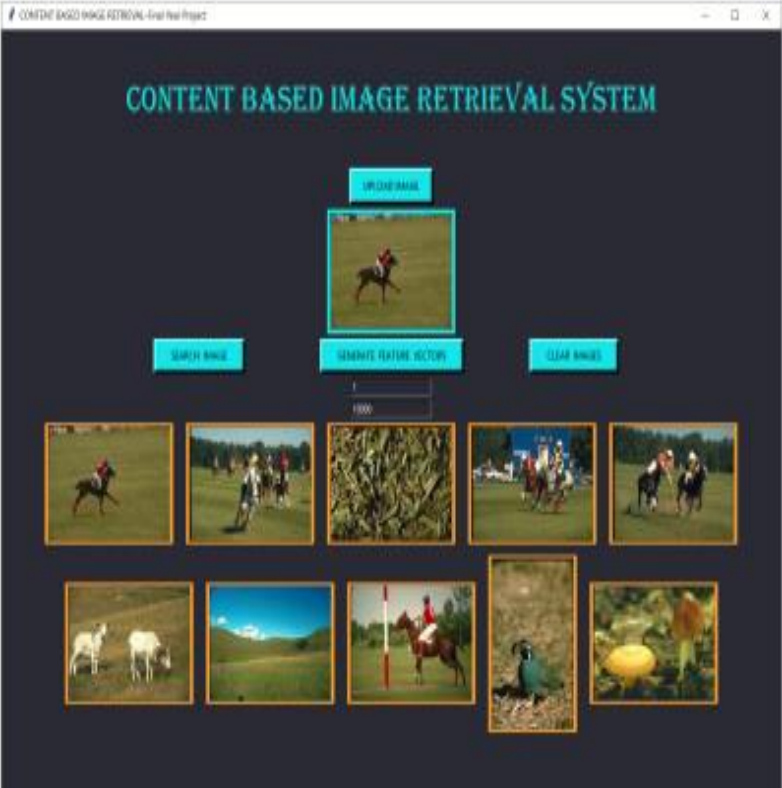

Fig 6. Relevant images for "Horsemen" category

Published By:

Blue Eyes Intelligence Engineering \& Sciences Publication 2303 (C) Copyright: All rights reserved.

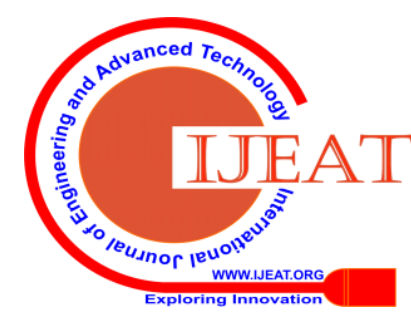


Table 1: Performance analysis of different categories of pictures

\begin{tabular}{|c|c|c|c|}
\hline S.No & Category & Existing Accuracy & $\begin{array}{r}\text { Proposed } \\
\text { Accuracy }\end{array}$ \\
\hline 1 & Paintings & $76 \%$ & $81 \%$ \\
\hline 2 & Planets & $72 \%$ & $85 \%$ \\
\hline 3 & Lions & $50 \%$ & $55 \%$ \\
\hline 4 & Mountains & $63 \%$ & $75 \%$ \\
\hline 5 & Martial Arts & $80 \%$ & $95 \%$ \\
\hline 6 & Clouds & $27 \%$ & $30 \%$ \\
\hline \multicolumn{2}{|c|}{$\begin{array}{c}\text { Total (Average for } 99 \\
\text { Categories) }\end{array}$} & $65 \%$ & $77 \%$ \\
\hline
\end{tabular}

\section{CONCLUSION}

In this work, picture similarity to the color histogram was used. We used segmentation, and from all regions, produced Local Color Histograms (LCH). Again the normalization of histograms was used to deal with variations in pixel counts of identical images of different sizes. The optimal configuration in the histogram is tried for the number of $\mathrm{H}, \mathrm{S}$ and $\mathrm{V}$ bins, and a bin configuration of $(16,7$, and 7$)$ worked well. We have tried to find a reasonable way of segmenting the picture into different local regions. Since the subject appears to be in the center of the picture in most of the pictures,and held a specific local area (the ellipse) in the middle. With experiments we find that 20 percent of the corresponding picture side would be the length of its axes

\section{REFERENCES}

1. Munjal MN, Bhatia S. A Novel Technique for Effective Image Gallery Search using Content Based Image Retrieval System. In2019 International Conference on Machine Learning, Big Data, Cloud and Parallel Computing (COMITCon) 2019 Feb 14 (pp. 25-29). IEEE.

2. Dai OE, Demir B, Sankur B, Bruzzone L. A novel system for content-based retrieval of single and multi-label high-dimensional remote sensing images. IEEE Journal of Selected Topics in Applied Earth Observations and Remote Sensing. 2018 Jun 21;11(7):2473-90.

3. Dash JK, Mukhopadhyay S, Gupta RD. Content-based image retrieval using fuzzy class membership and rules based on classifier confidence. IET Image Processing. 2015 Sep 1;9(9):836-48.

4. Jiao L, Tang X, Hou B, Wang S. SAR images retrieval based on semantic classification and region-based similarity measure for earth observation. IEEE journal of selected topics in applied earth observations and remote sensing. 2015 May 22;8(8):3876-91.

5. Guo JM, Prasetyo H. Content-based image retrieval using features extracted from halftoning-based block truncation coding. IEEE Transactions on image processing. 2014 Nov 20;24(3):1010-24.

6. Ramos J, Kockelkorn TT, Ramos I, Ramos R, Grutters J, Viergever MA, van Ginneken B, Campilho A. Content-based image retrieval by metric learning from radiology reports: application to interstitial lung diseases. IEEE journal of biomedical and health informatics. 2014 Nov 25;20(1):281-92.

7. Sevilla J, Jiménez LI, Plaza A. Sparse unmixing-based content retrieval of hyperspectral images on graphics processing units. IEEE Geoscience and Remote Sensing Letters. 2015 Oct 19;12(12):2443-7.

8. Guo JM, Prasetyo H, Chen JH. Content-based image retrieval using error diffusion block truncation coding features. IEEE Transactions on Circuits and Systems for Video Technology. 2014 Sep 15;25(3):466-81.

9. Ma Y, Jiang Z, Zhang H, Xie F, Zheng Y, Shi H, Zhao Y. Breast histopathological image retrieval based on latent dirichlet allocation. IEEE journal of biomedical and health informatics. 2016 Sep 20;21(4):1114-23.

10. Wei S, Liao L, Li J, Zheng Q, Yang F, Zhao Y. Saliency Inside: Learning Attentive CNNs for Content-Based Image Retrieval. IEEE Transactions on Image Processing. 2019 May 2;28(9):4580-93.

\section{AUTHORS PROFILE}

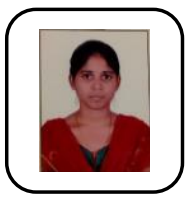

Madhuri Pydi Studying Master of Technology, Department Of Computer Science and Engineering, Velagapudi Ramakrishna Engineering College, Vijayawada. Obtained B.Tech Degree in Computer Science and Engineering in NRIIT Agiripalli, Vijayawada, Andhra Pradesh, India, in 2018.

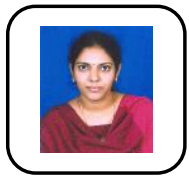

K.L.Sailaja received her B.Tech (CSE), M.Tech (CSE). She is currently working as Assistant Professor in VR Siddhartha Engineering College in Department of Computer Science and Engineering Vijayawada, Andhra Pradesh. Her Research interest included Digital Image Processing and Network Security.

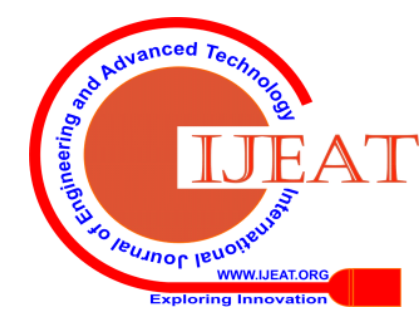

